\title{
The Effect of the Chemotherapy Period on the Fatigue Symptom in Gynecological Cancer Patients
}

\author{
Hacer Alan Dikmen, Department of Midwifery, Faculty of Health Sciences, Selcuk University
}

\begin{abstract}
Problem Statement: Gynecological cancer is in the first place in the cancers in women in Turkey and in the world, the number of cases increases every year. In gynecological cancers, chemotherapy is the most frequently used treatment option after surgery in spite of its side effects. In gynecological cancer patients, the most common side effect of chemotherapy is the fatigue symptom which affects the patients' quality of life.

Method: This review was written by evaluating the findings of 17 articles on cancer and fatigue which were published between 2000 and 2012. The articles were searched in online data bases between 01.07.2017 and 20.08.2017. The aim of this study is to discuss the effect of the chemotherapy period on the fatigue symptom in gynecological cancer patients.

Results: In the period of cancer treatment, anemia, cachexia, fever, infections, metabolic diseases, sedative medications, obesity, low activity level, alcohol use, environmental toxins, previously received cancer treatments are among the most important causes of fatigue. It is also known that women are more likely to experience the fatigue symptom in cancer treatment than men. Most of the patients (80-99\%) experience fatigue symptoms during chemotherapy. Fatigue symptoms may continue in $15-40 \%$ of the patients even after years of treatment. In addition, there are studies showed that fatigue is permanent in $17-26 \%$ of the cases. The insomnia due to menopausal symptoms in the women who underwent oophorectomy also leads to fatigue. The side effects regarding the gastrointestinal system which are developed during chemotherapy and especially fatigue resulting from anemia due to bone marrow depression may negatively affect womens` quality of life.

Conclusion: Fatigue is the most common symptom in gynecological cancer patients during chemotherapy and it negatively affects the patients' quality of life. Healthcare professionals should determine the cause of fatigue at first and develop strategies for coping with the fatigue symptom that develop in the patients using pharmacological or non-pharmacological methods. If the patients have anemia, fever, infection, dehydration, electrolyte imbalances, cachexia, depression, anxiety, pain, sleep problems and hypothyroidism, appropriate treatment and care should be applied. The training should be given to protect and increase the energy of the patients.
\end{abstract}

Key Words: gynecological cancer, chemotherapy, fatigue

References

Cella, D. F., Lai, J. S., Chang, C. H., Peterman, A., \& Slavin, M. (2002). Fatigue in cancer patients compared with fatigue in the general United States population. Cancer, 94(2), 528-538.

Ding, Y., Zhu, Y. L., \& Zhang, M. F. (2007). Quality of life of chinese patients with ovarian malignancies during chemotherapy under condition of no recurrence. Cancer Nursing, 30(3), 243-250.

Holzner, B., Kemmler, G., Meraner, V., Maislinger, A., Kopp, M., Bodner, T., et al. (2003). Fatigue in ovarian carcinoma patients: a neglected issue? Cancer, 97(6), 1564-1572.

Mitchell, S.A., Hoffman, A.J., Clark, J. C., DeGennaro, R. M., Poirier, P., Robinson, C. B. Et al. (2014). Putting evidence into practice: an update of evidence-based interventions for cancer-related fatigue during and following treatment. Clinical Journal of Oncology Nursing, 18(6), 38-58. Pizarro, C. L., Gich, I., Barthe, E., Rovirosa, A., Farrus, B., Casas, F., et al. (2007). A randomized trial of the effect of training in relaxation and guided imagery techniques in improving psychological and quality-of-life indices for gyneclologic and breast brachytherapy patients. PsychoOncology, 1(16), 971-979.

Radbruch, L., Strasser, F., Elsner, F., Gonçalves, J. F., Loge, J., Kaasa, S., et al. (2008). Fatigue in palliative care patients-an EAPC approach. Palliative Medicine, 22(1), 13-32.

Steele, R., \& Fitch, M. I. (2008). Supportive care needs of women with gynecologic cancer. Cancer Nursing, 31(4), $284-291$.

Yamagishi, A., Morita, T., Miyashita, M., \& Kimura, F. (2009). symptom prevalence and longitudinal follow-up in cancer outpatients receiving chemotherapy. Journal of Pain and Symptom Management, 37(5), 823-830.

Copyright $\odot 2017$ Author Names and Contact Details Sadler, I. J., Jacobsen, P. B., Booth-Jones, M., Belanger, H., Weitzner, M. A., \& Fields, K. K. (2002). Preliminary evaluation of a clinical syndrome approach to assessing cancer-related fatigue. Journal of Pain and Symptom Management, 23(5), 406-416. 